\title{
Эффективность ценообразования российских IPO
}

\author{
Ивашковская И.В. ${ }^{21}$, Харламов Л.С. ${ }^{22}$
}

B статье анализируются результаты IPO, проведенных российскими компаниями с 1996 г. по I квартал 2007 г., и пути повышения эффективности их ценообразования. Решение данной задачи тесно связанно с понятием недооченки IPO (Underpricing) и так называемым феноменом частичного приспособления, четко прослеживающимся на данных по российским компаниям. Для оценки эффективности проведенного IPО обычно используют два подхода: сравнивают цену размещения с ценовым диапазоном (чем ближе к верхней границе ценового диапазона, тем лучше) либо с рыночной иеной по итогам первого дня торгов (недооченка IPO). Упомянутый ранее феномен частичного приспособления показывает, что чем ближе к верхней границе ценового диапазона оказалась цена размещения, тем выше недооченка IРО. Таким образом, возникает противоречие двух трактовок эффективности ценообразования. $B$ смягчении данного противоречия заключается задача поиска путей повышения эффективности ценообразования IPO (Initial Public Offering.

\section{Феномен частичного приспособления}

На сегодняшний день более 40 российских компаний провели IPO, что позволяет изучать формирующийся национальный опыт. Одним из ключевых направлений исследования российских IPO является оценка их эффективности. Эффективность IPO можно определять в сравнении с альтернативными способами привлечения капитала, например размещением по закрытой подписке (private placement), либо с некой эталонной величиной, например с равновесным рыночным курсом акций компании. В данной статье речь пойдет о втором из предложенных вариантов.

В западной академической литературе, посвященной тематике IPO, выявлен ряд стилизованных фактов относительно ценообразования IPO, одним из которых является недооценка IPO (IPO Underpricing). По оценкам западных специалистов, в среднем в процессе IPO курс размещения несколько ниже курса закрытия первого дня торгов: например, в США по совокупным данным на протяжении почти всего 20 века отмеченная разница курсов составляла порядка 15\% [Ritter, 1998]. Похожее явление существует и в механизмах российских IPO, как показано в таблице 1, где представлены данные по недооценке IPO, осуществленных российскими компаниями в целом, а также в разбивке по стране размещения. Как видно из таблицы, недооценка IPO российских компаний пока значительно ниже, чем 15\%, характерных для данных по американским компаниям.

Таблица 1. Недооценка IPO российских компаний по данным за 1996 г. І кв. 2007 г. в разбивке по стране размещения и в целом по выборке

\begin{tabular}{|c|c|c|c|}
\hline Россия & Великобритания & США & Вся выборка \\
\hline \hline $4,98 \%$ & $3,26 \%$ & $8,72 \%$ & $4,78 \%$ \\
\hline \hline
\end{tabular}

Источник: собственные расчеты

\footnotetext{
${ }^{21}$ Канд. экон.наук, профессор, заведующая кафедры экономики и финансов фирмы, заведующая лаборатории корпоративных финансов факультета экономики ГУ ВШЭ.

${ }^{22}$ Магистр экономики, сотрудник научно-учебной лаборатории корпоративных финансов ГУ-ВШЭ, кредитный аналитик Международного Московского Банка.
}

Выпуск \#3, 2007 С Электронный журнал Корпоративные Финансы, 2007 
С другой стороны, об эффективности IPO судят и по соотношению курса размещения и ранее установленного ценового диапазона: чем ближе цена размещения к верхней границе ценового диапазона, тем более успешным считается IPO.

Наконец, необходимо учитывать и еще один ценовой ракурс: по результатам многочисленных исследований доказано, что чем выше в среднем курс размещения по сравнению с серединой цеенового диапазона, тем выше недооценка ІРО. Это явление принято называть феноменом частичного приспособления (partial adjustment phenomenon). Чтобы его наблюдать, исследователи изучают взаимосвязь двух показателей: недооценки IPO (IPO underpricing) и индекса изменения цены размещения (price revision index) [Hanley, 1993].

На данных по российским компаниям существование феномена частичного приспособления также подтверждается.

На рис. 1 по вертикали отложены данные о величине недооценки IPO:

$$
U N=(P c 1-P o) / P_{O}
$$

где Рc1 - цена закрытия первого дня торгов, Ро - цена первичного размещения.

По горизонтали: индекс изменения цены размещения по сравнению с серединой ценового диапазона (price revision index), рассчитанный в соответствии с формулой:

где $P e=(P h+P l) / 2$,

$$
P R I=(P o-P e) / P e
$$

Ро - цена первичного размещения,

$\mathrm{Ph}$ - верхняя граница ценового диапазона,

$\mathrm{Pl}$ - нижняя граница ценового диапазона.

\section{Рис. 1. Феномен частичного приспособления на данных по IPO российских компаний за период 1996 г. — I кв. 2007 г.}

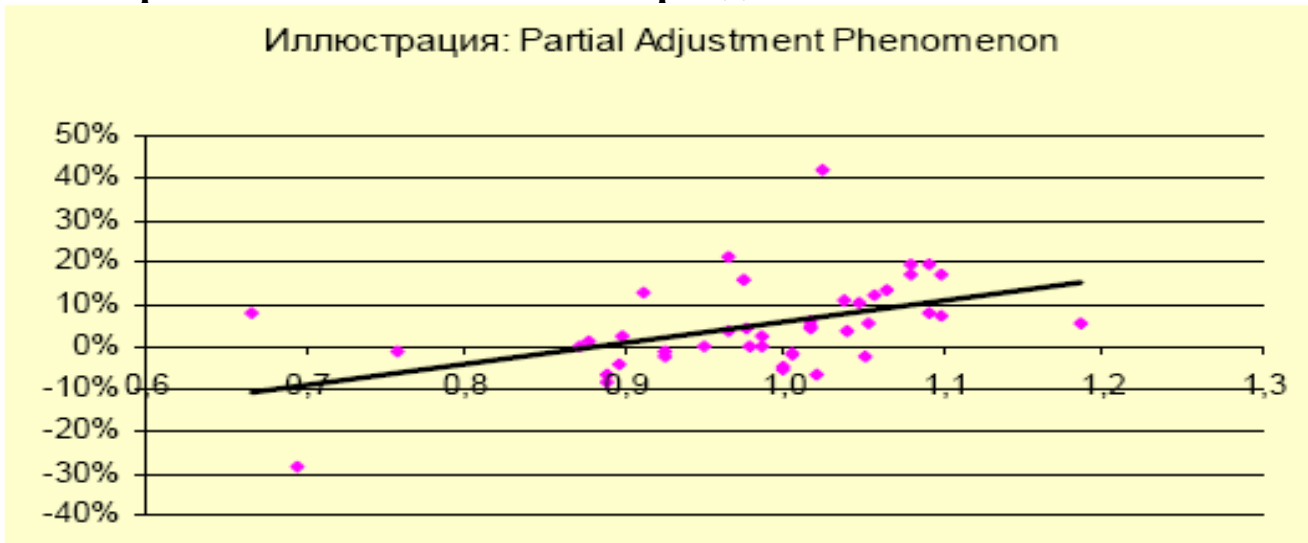

Источник: собственные расчеты

Из рис. 1 следует, что чем выше цена размещения акиий российских компаний по сравнению с серединой ценового диапазона (по горизонтали), тем выме величина недооченки IPO (по вертикали).

По сути, мы получаем противоречие между первой трактовкой эффективности ценообразования IPO (на основе соотношения с ценовым диапазоном) и второй (соотношения с рыночной ценой по итогам первого дня торгов, недооенка IPO). 
Теоретическое обоснование существования феномена частичного приспособления впервые дано в работе Бенвенисте и Спиндта [Benveniste\&Spindt, 1989], где авторы смоделировали процесс ценообразования IPO методом книги заявок. Данный феномен описывается авторами с помощью полученных результатов:

- Организатор в процессе заполнения книги заявок пытается извлечь позитивную информацию по поводу максимальной цены, которую покупатели готовы дать за акции эмитента.

- Чем больше позитивной информации будет извлечено в процессе заполнения книги заявок, тем выше будет цена размещения по сравнению с серединой ценового диапазона.

- Инвесторы не заинтересованы в раскрытии информации о готовности платить, так как это увеличит цену размещения, и каждый конкретный инвестор извлечет меньшую выгоду из покупки акций эмитента.

- Организатор вынужден мотивировать инвесторов раскрывать честно информацию. Инструментами мотивации выступают количество акций, передаваемых конкретному инвестору и цена размещения.

- Смысл мотивации количеством акций заключается в том, что чем выше цена, которую готов отдать инвестор, тем большую долю эмиссии получает данный инвестор. Однако в случае, когда количество инвесторов, готовых платить высокую цену, становится слишком большим, данный инструмент себя исчерпывает и организатор начинает манипулировать ценой размещения.

- Смысл мотивации ценой размещения заключается в том, что в случае большого интереса со стороны инвесторов к размещению организатор лишь частично приспосабливает цену размещения к растущему спросу на акции компании. В этом и заключается суть феномена частичного приспособления.

В исследовании, представленном в данной статье, Зависимость, составляющая суть феномена частичного приспособления на данных по российским компаниям, является одной из самых тесных. В выборке из 43 наблюдений (описание выборки дано ниже), результаты оценки регрессии следующие ${ }^{23}$ :

Таблица 2. Результаты оценки регрессии, характеризующей феномен частичного приспособления на данных по IPO российских компаний в период с 1996 г. по I кв. 2007 г.

\begin{tabular}{|c|c|c|c|c|c|}
\hline \multicolumn{2}{|c|}{ Regression Statistics } & \multicolumn{3}{|c|}{ Underpricing $=\mathrm{a}+\mathrm{b} *$ Price Revision Index } & \\
\hline Multiple R & 0,4765 & & & & \\
\hline R Square & 0,227 & & & & \\
\hline \multicolumn{6}{|l|}{ Adjusted $R$} \\
\hline Square & 0,2082 & & & & \\
\hline Observations & 43 & & & & \\
\hline \multicolumn{6}{|l|}{ ANOVA } \\
\hline & $\overline{D f}$ & $S S$ & $M S$ & $\mathrm{~F}$ & Significance F \\
\hline Regression & 1 & 0,1139 & 0,1139 & 12,048 & 0,0012 \\
\hline Residual & 41 & 0,3877 & 0,0094 & & \\
\hline \multirow[t]{3}{*}{ Total } & 42 & 0,5016 & & & \\
\hline & & Standard & & & \\
\hline & Coefficients & Error & $t$ Stat & P-value & \\
\hline Intercept & $-0,4447$ & 0,1426 & $-3,1169$ & 0,0033 & \\
\hline Price Revision & & & & & \\
\hline Index & 0,5023 & 0,1447 & 3,4710 & 0,0012 & \\
\hline
\end{tabular}

${ }^{23}$ Регрессия построена в программном пакете Microsoft Excel.

Выпуск \#3, 2007

() Электронный журнал Корпоративные Финансы, 2007 
Из таблицы 2 видно, что коэффициент при индексе изменения цены размещения равен 0,5 . Это означает, что при росте индекса на $1 \%$ недооценка в среднем увеличивается на $0,5 \%$. Если предположить, что рост недооценки и рост индекса произошли в результате увеличения равновесного рыночного курса, выявленного в процессе роад-шоу, то коэффициент приспособления цены первичного размещения к росту равновесного курса для российских компаний, по результатам исследования, составляет $2 / 3$. В этом и заключается частичность приспособления.

O качестве регрессии говорит статистическая значимость F-статистики на уровне $1 \%$. Коэффициент при индексе изменения цены размещения также значим на доверительном интервале $1 \%$. Показатель R-квадрат составил $23 \%$, в данном случае он эквивалентен показателю корреляции, то есть коэффициент корреляции между двумя показателями составил 0,23.

Отметим, что после введения понятия недооценки IPO стратегия повышения эффективности ценообразования IPO казалась простой: для повышения эффективности необходимо просто снизить величину недооценки. Между тем единственным на сегодняшний день методом размещения акций как в РФ, так и на всех других развивающихся рынках ${ }^{24}$ является метод книги заявок. Как было сказано выше, при размещении акций данным методом неизбежно возникает феномен частичного приспособления, который снижает эффективность ценообразования IPO. Несмотря на то что эмитенты и организаторы рапортуют об успешном размещении вблизи верхней ценовой границы, реальный выявленный в процессе роад-шоу спрос на акции компании позволяет установить справедливую цену значительно выше.

\section{Исследование путей повышения эффективности ценообразования IPO}

В контексте наблюдаемого конфликта интересным представляется изучение влияния различных факторов на величину недооценки IPO и на индекс изменения цены размещения. Нами представлены результаты исследования, в котором проведен детальный анализ влияния доступных для измерения факторов на данные переменные. Исследование проводилось с позиций эмитента, поэтому при анализе различных факторов решались следующие основные задачи:

1.Во-первых, изучались возможности смягчения феномена частичного приспособления. Для этой цели выявлялись факторы, оказывающие одновременно положительное влияние на индекс цены размещения и отрицательное влияние на недооценку IPO.

2.Во-вторых, изучались возможности получения эмитентом дополнительного дохода без ухудшения эффективности ценообразования IPO. По сути, изучались факторы, оказывающие положительное влияние на индекс и при этом никак не влиявшие на величину недооценки.

3.В-третьих, изучалось влияние факторов, характеризующих российскую специфику.

\section{Описание данных}

История российских IPО сравнительно короткая и начинается в 1996 году, когда сотовый оператор «Вымпелком» (владелец торговой марки «БиЛайн») разместил свои акции на Нью-Йоркской фондовой бирже (NYSE) и произвел

\footnotetext{
${ }^{24}$ На развитых рынках встречаются иные способы размещения, однако это бывает крайне редко. Выпуск \#3, 2007

(c) Электронный журнал Корпоративные Финансы, 2007
} 
фурор среди инвесторов, чей спрос на ценные бумаги компании во много раз превысил предложение. «Вымпелком» оказался также первым и по величине недооценки: в первый день вторичных торгов цена акций оператора превысила цену размещения более чем на $40 \%{ }^{25}$ !

Источниками данных по предложенным ценовым диапазонам, ценам первичного размещения, ценам закрытия 1-го дня вторичных торгов для исследования служили материалы The PBN Company под названием «Российские пионеры IPO», находящиеся в свободном доступе на сайте компании (http://www.pbnco.com), где представлены данные по 23 российским IPO, проведенным вплоть до конца 2005 года. Для сбора данных по остальным размещениям были использованы информационные порталы http:/quote.ru uhttp://www.ipo-congress.ru . Информация по котировкам на вторичных торгах (цена открытия/закрытия первого дня торгов) по всем без исключения эмитентам была собрана в информационной базе Bloomberg. ${ }^{26}$ В итоге полный набор данных был собран по 39 компаниям. В выборку не попали: IMS Group, Группа «Черкизово» (данные по торгам в РФ), а также компания «Белон». По всем перечисленным компаниям наблюдалась низкая активность на вторичных торгах, так как момент начала вторичных торгов наступил значительно позже размещения (несмотря на то что торги были разрешены через $1-2$ дня после размещения).

Одной из важных предпосылок, сделанных в ходе построения выборки, является предположение о том, что зависимость между вторичными торгами на российских биржах и западных (Великобритания, США) в целом несущественна. Введение данной предпосылки позволило рассматривать совмещенные IPO (когда размещение проходило и в РФ, и в Великобритании/США) как независимые размещения. Это позволило увеличить выборку до 43 наблюдений (против 39 в противном случае). Проведенные впоследствии тесты показали, что от такого искусственного разделения качество выборки не поменялось. Среди компаний, оставшихся в выборке, нет инвестиционных фондов закрытого типа (closed-end funds), а также других компаний, чье ценообразование не подчиняется общим законам (таких, как Master Limited Partnerships, финансовые организации). Соответственно все оставшиеся компании были оставлены в выборке.

\section{Описание переменных}

В нашей модели использованы следующие зависимые переменные:

Недооценка IPO (Underpricing), или $\mathbf{U N}$, рассчитывается по следующей формуле:

$$
U N=(P c 1-P o) / P o,
$$

где Pc1 - цена закрытия первого дня торгов, Ро - цена первичного размещения.

RI - процентное изменение цены первичного размещения по сравнению с ожидаемой ценой первичного размещения (revision index). Оно характеризует соотношение иены размещения с заявленным иеновым диапазоном и в разных регрессиях выступает как зависимая и как независимая переменная. Формула (4) для расчета представлена ниже:

\footnotetext{
${ }^{25}$ К апрелю 2007 г. российские компании провели уже 42 IPO на американских, лондонских, а также российских площадках.

26 Доступ к терминалу Bloomberg приобретен в рамках Инновационной образовательной программы ГУ ВШЭ.
} 
(4)

$$
R I=(P o-P e) / P e, \text { где } P e=(P h+P l) / 2,
$$

где Ро - цена первичного размещения,

$\mathrm{Ph}$ - верхняя граница ценового диапазона,

$\mathrm{Pl}$ - нижняя граница ценового диапазона.

В некоторых спецификациях моделей представлен также показатель PRI, рассчитываемый по формуле 5. Смысл этого показателя тот же, что и в предыдущем примере.

$$
P R I=P o / P e .
$$

Использование PRI вместо RI связано с невозможностью логарифмирования отрицательных значений RI.

B качестве независимых переменных взяты следующие показатели:

WPR - иирина иеенового диапазона в прочентном выражении (width of price range). Данный показатель характеризует уровень еx ante риска эмиссии, как было уже описано выше. Расчет данного регрессора проводился по следующей формуле:

$$
W P R=(P h-P l) / P e^{.}
$$
1993].

Данный регрессор также был предложен в исследовании Хэнли [Hanley,

AIE - ожидаемый объем выпуска (amount of issue expected). Аналогично данный регрессор характеризует меру ex ante риска эмиссии. Формула для расчета:

$$
A I E=(Q-Q o p) * P e,
$$

где $\mathrm{Q}$ - итоговое количество размещенных акций,

Qop - количество акций, размещенных путем использования опциона на доразмещение. 1993].

Данный регрессор также был предложен в исследовании Хэнли [Hanley,

В целом значимость ех ante риска размещения предсказывается в модели Benveniste-Spindt: чем выше ex ante риск, тем выше предельная полезность «положительной» информации.

RTS15 - прочентное изменение индекса РТС за последние 15 рабочих сессий до момента размещения. Данный регрессор характеризует конъюнктуру рынка непосредственно перед размещением. Для целей исследования также будет использоваться показатель RTS30, однако ожидается, что RTS15 является более точным показателем конъюнктуры, так как заполнение книги заявок в конкретном размещении вступает в активную фазу только за несколько рабочих сессий до назначенной даты первичного размещения. На протяжении всего периода, пока идет роад-шоу, большинство инвесторов занимает выжидательную позицию. Формула для расчета фактора:

$$
R T S 15=\left(R T S_{0} / R_{-15}\right)-1,
$$

где $\mathrm{RTS}_{0}$ означает значение индекса РТС в день первичного размещения.

RTS $_{-15}$ означает значение индекса РТC за 15 рабочих сессий биржи РТС до первичного размещения конкретной эмиссии. Формула для расчета RTS30 аналогичная. В исследовании Хэнли [Hanley, 1993] был предложен аналогичный регрессор на основе индекса NASDAQ.

LM1 - дамми-переменная, принимающая значение 1, когда размещение проходит в РФ, и 0 во всех других случаях. Данный регрессор введен для проверки гипотезы, отличается ли процесс ценообразования IPO на российских биржах от аналогичного процесса на развитых рынках. 
LM2 - дамми-переменная, принимающая значение 1 в случае, если одним из двух основных андеррайтеров является российский инвестбанк, и $0-$ в противном случае. Данная переменная введена для проверки точки зрения, утверждающей, что российские андеррайтеры лучше знают российский рынок и более внимательно подходят к каждому конкретному клиенту, что ведет к более высокому доходу эмитента. Существует также другая взаимоисключающая гипотеза: международные инвестбанки имеют большую долю на рынке, более широкую базу инвесторов, поэтому размещение под управлением международного инвестбанка более выгодно для эмитента.

\section{Результаты исследования}

Данное исследование предполагает использование линейных регрессий, оцененных методом наименьших квадратов, при построении факторных моделей. Bсе регрессии оценивались в программном пакете EViews5. Факторные модели с одними и теми же регрессорами строились поочередно для индекса изменения цены первичного размещения и затем для недооценки IPО. При построении всех регрессий стандартные ошибки данных регрессий корректировались для нейтрализации возможной гетероскедастичности методом Уайта.

Результаты на совокупной выборке показаны в следующей таблице.

Таблица 3. Взаимосвязь RI и UN. Совокупная выборка

\begin{tabular}{|c|c|c|c|c|}
\hline & \multicolumn{2}{|c|}{$\operatorname{Ln}(\mathrm{PRI})$} & \multicolumn{2}{|c|}{$\mathbf{U N}$} \\
\hline & \multicolumn{2}{|c|}{ Совокупная выборка } & \multicolumn{2}{|c|}{ Совокупная выборка } \\
\hline & t-stat. & p-value & t-stat. & $p$-value \\
\hline Константа & \multicolumn{2}{|c|}{ - } & \multicolumn{2}{|c|}{ - } \\
\hline \multirow[t]{2}{*}{$\mathbf{R I}$} & \multicolumn{2}{|c|}{ - } & \multicolumn{2}{|c|}{$\mathbf{0 , 5 5 7 7}$} \\
\hline & & & 2,9505 & 0,0053 \\
\hline \multirow[t]{2}{*}{ WPR/Ln (WPR) } & \multicolumn{2}{|c|}{$-0,2411$} & \multicolumn{2}{|c|}{$-0,0168$} \\
\hline & $-1,9057$ & 0,0639 & $-1,6917$ & 0,0985 \\
\hline AIE/Ln (AIE) & \multicolumn{2}{|c|}{0,0154} & \multicolumn{2}{|c|}{$-0,0221$} \\
\hline & 1,9246 & 0,0614 & $-3,3594$ & 0,0017 \\
\hline RTS30 & \multicolumn{2}{|c|}{0,5635} & \multicolumn{2}{|c|}{ - } \\
\hline & 2,6827 & 0,0106 & & \\
\hline LM1 & \multicolumn{2}{|r|}{ 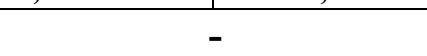 } & \multicolumn{2}{|c|}{-} \\
\hline LM2 & \multicolumn{2}{|c|}{-} & \multicolumn{2}{|c|}{-} \\
\hline R-квадрат & \multicolumn{2}{|c|}{$27,2 \%$} & \multicolumn{2}{|c|}{$32,9 \%$} \\
\hline
\end{tabular}

Наибольший интерес представляют переменные, оказавшиеся значимыми в обеих регрессиях на совокупной выборке. Таких на выборке оказалось только две: ожидаемый объем эмиссии (AIE) и ширина ценового диапазона (WPR). Напомним, что обе переменные характеризуют неопределенность относительно цены размещения.

Коэффициенты при WPR в обеих регрессиях имеют одинаковый знак, что говорит о противоречивости полученного результата. Отрицательный коэффициент при WPR в модели изменения цены размещения (Ln (PRI)), как уже упоминалось выше, свидетельствует о том, что более рискованные размещения пользуются меньшим спросом у инвесторов. При этом отрицательный 
коэффициент при WPR в регрессии UN говорит о том, что величина недооценки более рискованных IPO несколько ниже, что противоречит выводам Бенвенисте и Спиндта [Benveniste\&Spindt, 1989], показавшим, что чем более высока неопределенность относительно цены размещения, тем выше предельная полезность информации, полученной в процессе роад-шоу, и тем большей должна быть компенсация в виде роста котировок на вторичном рынке (недооценки). Однако отметим, что по абсолютной величине коэффициент при WPR в регрессии близок к нулю и значим только на 10\%-ном уровне значимости. Таким образом, полученный результат не бесспорен.

Коэффициенты при AIE, наоборот, не противоречат друг другу и подтверждают выводы более ранних исследований. Положительный знак коэффициента в модели изменения цены размещения (Ln (PRI)) говорит о том, что менее рискованные IPO в целом пользуются большим интересом/спросом инвесторов. При этом менее рискованные IPO характеризуются меньшей предельной полезностью позитивной информации во время роад-шоу, что ведет к меньшей недооценке, что и следует из регрессии UN. Эмпирически аналогичный результат также получили Риттер [Ritter, 1987] и Хэнли [Hanley, 1993]. Теоретической базой, как и во всех других примерах, служит модель Бенвенисте и Спиндта.

Отметим, что другие регрессоры при анализе факторов недооценки IPO (UN) оказались незначимы на совокупной выборке. Что касается факторов интереса инвесторов к размещению (RI, PRI), то значимой оказалась также конъюнктура российского рынка акций в период роад-шоу (RTS30). Отметим, что у Хэнли на данных по рынку США фактор, аналогичный RTS30, был значимым также в модели недооценки.

Остались незначимыми для обеих регрессий переменные LM1, LM2. Таким образом, гипотезы о влиянии на интерес инвесторов и величину недооценки страны размещения и страны происхождения главных организаторов не подтвердились.

Резюмируя полученные результаты, отметим, что с точки зрения эмитента важным фактором для максимизации дохода от размещения может оказаться положительная конъюнктура рынка в процессе роад-шоу. Кроме того, при принятии решения об IPO эмитентам (особенно небольшим по размеру) следует задуматься о максимизации продаваемой доли капитала. Как показали результаты исследования, рост объема IPO ведет к росту цены первичного размещения при одновременном сокращении недооценки. На сегодняшний день наибольшим интересом у инвесторов пользуются размещения объемом примерно 1 млрд руб. и выше.

Отметим также, что объем IPO характеризует неопределенность относительно цены размещения. Вероятным способом снижения недооценки (и неопределенности), а также увеличения цены первичного размещения может стать выжидательная позиция: когда IPO провели уже несколько аналогов, неопределенность относительно цены (и величина недооценки) резко падает. К подобным выводам в своих исследованиях пришли: Бенвенисте, Бусаба и Вильхельм [Benveniste, Busaba and Wilhelm, 2002], Бенвенисте, Лжунгквист, Вильхельм и Ю [Benveniste, Ljungqvist, Wilhelm and $\mathrm{Yu}, 2003$ ]. Они также показали, что возможным решением проблемы «первопроходца» может быть обращение к андеррайтеру, специализирующемуся на отрасли эмитента.

Не уменьшая важность полученных выводов, важно отметить, что согласно $\mathrm{R}$-квадрат полученные модели описывают поведение зависимых переменных не более чем на треть. По сути, мы до сих пор, возможно, не знаем других ключевых 
факторов, влиявших на интерес инвесторов/недооценку, либо, что более вероятно, эти факторы просто невозможно измерить.

Таким образом, важен более глубокий анализ выборки, для чего она была разделена на две условные части: Upper revisions (размещения, пользовавшиеся интересом инвесторов; цена размещения выше середины ценового диапазона) и Lower revisions (размещения, не пользовавшиеся интересом инвесторов; цена размещения ниже или равна середине ценового диапазона). Смысл разделения выборки на эти две части заключается в том, что если мы не смогли найти факторы изменения индекса цены размещения на совокупной выборке (на 5\%-ном уровне значим только RTS30, который к тому же совершенно неподконтролен), то логично будет искусственно разделить выборку на размещения, пользовавшиеся интересом инвесторов, и на все остальные.

Ниже представлен результат оценки регрессий на выборке Upper revisions.

Таблица 4. Взаимосвязь RI и UN. Выборка Upper Revisions

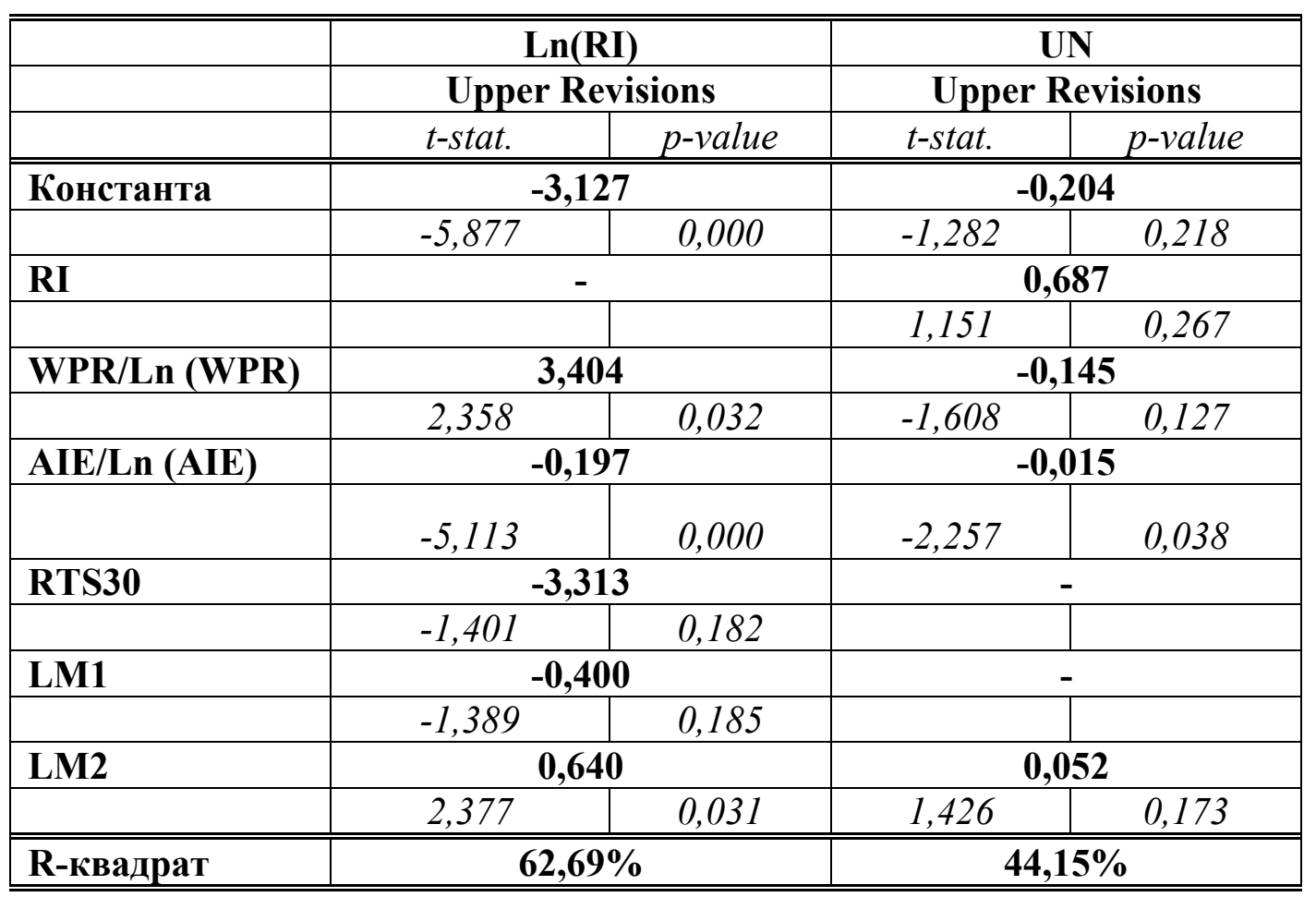

Отметим, что на данной выборке значительно большее количество факторов оказалось значимым. Предсказательная сила модели Ln (RI) несколько завышена, так как на её качество сильное влияние оказало IPO «Роснефти», объем которого более чем в 10 раз превышал средний показатель. В итоге коэффициент при показателе объема IPO (AIE) в модели оказался наиболее значимым.

Важнейтей находкой в процессе анализа факторной модели RI является факт, что на выборке Upреr revisions более высоким интересом инвесторов пользовались размещения с умеренным риском, нежели с минимальным, о чем говорит знак коэффициента при WPR. Другой находкой является факт, что в выборке пользовавшихся интересом инвесторов размещений более высокий интерес инвесторы проявляли $\kappa$ размещениям, где одним из главных организаторов являлся российский инвестиционный банк.

Отдельно был проведен анализ поведения инвесторов во время первичного размещения и вторичных торгов на выборке, которая характеризовалась в целом низким интересом инвесторов к размещению. Результат показан в таблице 5. 
Таблица 5. Взаимосвязь RI и UN. Выборка Lower Revisions

\begin{tabular}{|c|c|c|c|c|}
\hline & \multirow{2}{*}{\multicolumn{2}{|c|}{$\begin{array}{c}\text { Ln (PRI) } \\
\text { Lower revisions } \\
\end{array}$}} & \multirow{2}{*}{\multicolumn{2}{|c|}{$\begin{array}{c}\text { UN } \\
\text { Lower revisions } \\
\end{array}$}} \\
\hline & & & & \\
\hline & t-stat. & $p$-value & t-stat. & $p$-value \\
\hline \multirow[t]{2}{*}{ Константа } & \multicolumn{2}{|c|}{-} & \multicolumn{2}{|c|}{0,0875} \\
\hline & & & 2,3580 & 0,0292 \\
\hline \multirow[t]{2}{*}{ RI } & \multicolumn{2}{|c|}{ - } & \multicolumn{2}{|c|}{$\mathbf{0 , 4 5 3 7}$} \\
\hline & & 1,2763 & 0,2172 \\
\hline \multirow[t]{2}{*}{ WPR/Ln (WPR) } & \multicolumn{2}{|c|}{$-0,4380$} & \multicolumn{2}{|r|}{ 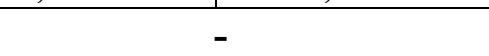 } \\
\hline & $-2,6034$ & 0,0175 & & \\
\hline \multirow[t]{2}{*}{ AIE/Ln (AIE) } & \multicolumn{2}{|c|}{$\mathbf{0 , 0 1 7 2}$} & \multicolumn{2}{|c|}{$-0,1363$} \\
\hline & 1,4886 & 0,1530 & $-3,5609$ & 0,0021 \\
\hline \multirow[t]{2}{*}{ RTS30 } & \multicolumn{2}{|c|}{0,3871} & \multicolumn{2}{|c|}{-} \\
\hline & 1,3067 & 0,2069 & & \\
\hline LM1 & \multicolumn{2}{|c|}{-} & \multicolumn{2}{|c|}{-} \\
\hline LM2 & \multicolumn{2}{|c|}{-} & \multicolumn{2}{|c|}{-} \\
\hline & & & & \\
\hline R-квадрат & \multicolumn{2}{|c|}{$25,67 \%$} & \multicolumn{2}{|c|}{$26,29 \%$} \\
\hline
\end{tabular}

Результаты, полученные на данной выборке, аналогичны совокупной: важно влияние риска, присущего размещению, и конъюнктуры фондового рынка. Таким образом, эмитенту, знающему заранее о том, что акции его компании не будут пользоваться очень высоким спросом инвесторов, для максимизации дохода от IPO важно угадать конъюнктуру рынка во время роад-шоу (однако ввиду низкой статистической значимости коэффициента при соответствующей переменной не будем преувеличивать значение этого фактора), а также постараться максимизировать объем IPO путем увеличения продаваемой доли капитала. Об альтернативных мерах по снижению неопределенности было сказано выше.

\section{Заключение}

В исследовании проведен комплексный анализ эффективности ценообразования IPO российских компаний. Были использованы данные по всем российским IPO за период с 1996г. по I квартал 2007 г. В контексте поставленных в рамках исследования задач получены следующие результаты:

1. Важным фактором, позволяющим повысить эффективность ценообразования IPO, является объем размещения. На сегодняшний день наибольшим интересом у инвесторов пользуются размещения объемом около 1 млрд руб. и выше.

2. Благоприятная конъюнктура фондового рынка накануне IPO позволяет эмитенту получить дополнительный доход в ходе IPO, не влияя на величину недооценки, что в целом также повышает эффективность размещения.

3. Среди заведомо привлекательных для инвесторов IPO привлечение российского инвестбанка в качестве одного из основных организаторов также позволяет эмитенту получить дополнительный доход, не влияя на недооценку. Данную находку можно трактовать как особенность российского рынка. 
4. Наиболее привлекательны для инвесторов IPO, характеризующиеся умеренным, а не минимальным уровнем неопределенности относительно цены размещения.

Полученные результаты позволяют лучше понять процесс ценообразования IPO и выявить пути повышения эффективности ценообразования российских IPO.

\section{Список литературы}

1. Гвардин, С. В. ІРО: стратегия, перспективы и опыт российских компаний. Москва: Вершина, 2007.

2. Ritter, J.R. Initial Public Offerings 1998 работа доступна в сети Интернет: http://bear.cba.ufl.edu/ritter.

3. Benveniste, Lawrence M. and Paul A. Spindt, 1989, How investment bankers determine the offer price and allocation of new issues, Journal of Financial Economics 24, 343-361.

4. Benveniste, L.M., W.Y. Busaba, and W.J. Wilhelm, Jr., 2002, Information Externalities and the Role of Underwriters in Primary Equity Markets, Journal of Financial Intermediation 11, 61-86.

5. Benveniste, L.M., A. Ljungqvist, W.J. Wilhelm, Jr., and X. Yu, 2003, Evidence of Information Spillovers in the Production of Investment Banking Services, Journal of Finance 58, 577-608.

6. Hanley, K., 1993, Underpricing of Initial Public Offerings and the Partial Adjustment Phenomenon, Journal of Financial Economics 34, 231-250.

7. Ritter, Jay R., 1987, The costs of going public, Journal of Financial Economics 19, 269-281. 